\title{
CORRECTION
}

\section{Correction to: A Low Complexity Correlation Algorithm for Compressive Channel Estimation in Massive MIMO System}

\author{
Ruoyu Zhang ${ }^{1} \cdot$ Honglin Zhao ${ }^{1} \cdot$ Chengzhao Shan ${ }^{1} \cdot$ Shaobo Jia ${ }^{1}$
}

Published online: 7 June 2018

(c) Springer Science+Business Media, LLC, part of Springer Nature 2018

\section{Correction to: \\ International Journal of Wireless Information Networks https://doi.org/10.1007/s10776-018-0398-z}

The original version of this article unfortunately contained a mistake in "Algorithm 1" under Section 3. The ellipsis in Algorithm 1 appears as alpha "L" in the published article. Hence, the corrected algorithm is presented with this erratum.

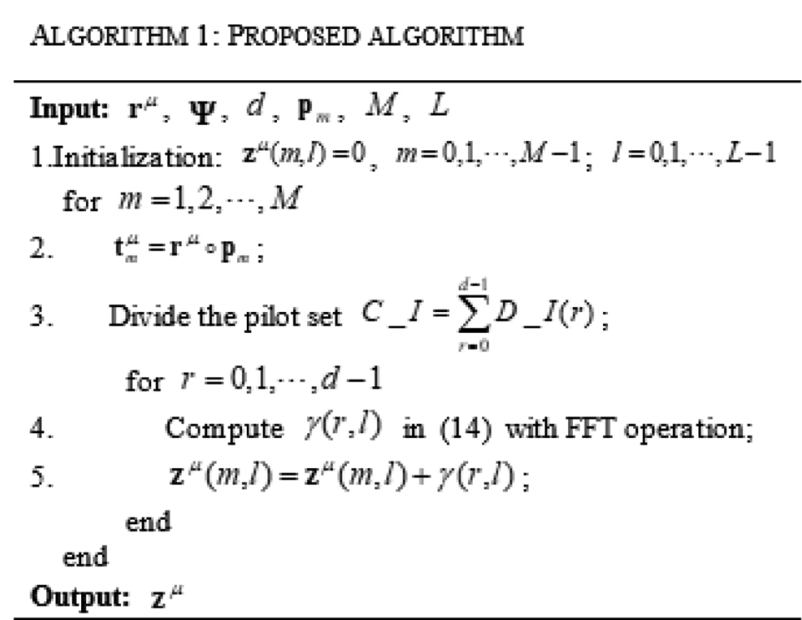

The original article has been corrected.
The original article can be found online at https://doi.org/10.1007/ s10776-018-0398-z.

Honglin Zhao

hlzhao@hit.edu.cn

Ruoyu Zhang

hitzhangruoyu@163.com

Chengzhao Shan

scz13384661358@sina.com

Shaobo Jia

jiashaobo2007@126.com

1 Communication Research Center, Harbin Institute of Technology, Harbin 150080, China 